\title{
Hybrid time-domain and continuous- wave diffuse optical tomography instrument with concurrent, clinical magnetic resonance imaging for breast cancer imaging
}

Jeffrey M. Cochran

David R. Busch

Li Lin

David L. Minkoff

Martin Schweiger

Simon Arridge

Arjun G. Yodh 


\title{
Hybrid time-domain and continuous-wave diffuse optical tomography instrument with concurrent, clinical magnetic resonance imaging for breast cancer imaging
}

\author{
Jeffrey M. Cochran, ${ }^{\mathrm{a}, \star}$ David R. Busch, ${ }^{\mathrm{a}, \mathrm{b}, \mathrm{c}, \mathrm{d}}$ Li Lin, ${ }^{\mathrm{a}, \mathrm{e}}$ David L. Minkoff, ${ }^{f}$ Martin Schweiger, ${ }^{\mathrm{g}}$ \\ Simon Arridge, ${ }^{9}$ and Arjun G. Yodh ${ }^{\mathrm{a}}$ \\ aUniversity of Pennsylvania, Department of Physics and Astronomy, Philadelphia, Pennsylvania, United States \\ bUniversity of Texas Southwestern Medical Center, Department of Anesthesiology and Pain Management, Dallas, Texas, United States \\ 'University of Texas Southwestern Medical Center, Department of Neurology and Neurotherapeutics, Dallas, Texas, United States \\ ${ }^{\mathrm{d} C h i l d r e n ' s ~ H o s p i t a l ~ o f ~ P h i l a d e l p h i a, ~ D e p a r t m e n t ~ o f ~ N e u r o l o g y, ~ P h i l a d e l p h i a, ~ P e n n s y l v a n i a, ~ U n i t e d ~ S t a t e s ~}$ \\ ${ }^{\mathrm{e} C}$ California Institute of Technology, Department of Medical Engineering, Pasadena, California, United States \\ fEmory University, Department of Medicine, Atlanta, Georgia, United States \\ 'University College London, Centre for Medical Image Computing, London, United Kigdom
}

\begin{abstract}
Diffuse optical tomography has demonstrated significant potential for clinical utility in the diagnosis and prognosis of breast cancer, and its use in combination with other structural imaging modalities improves lesion localization and the quantification of functional tissue properties. Here, we introduce a hybrid diffuse optical imaging system that operates concurrently with magnetic resonance imaging (MRI) in the imaging suite, utilizing commercially available MR surface coils. The instrument acquires both continuous-wave and time-domain diffuse optical data in the parallel-plate geometry, permitting both absolute assignment of tissue optical properties and three-dimensional tomography; moreover, the instrument is designed to incorporate diffuse correlation spectroscopic measurements for probing tissue blood flow. The instrument is described in detail here. Image reconstructions of a tissue phantom are presented as an initial indicator of the system's ability to accurately reconstruct optical properties and the concrete benefits of the spatial constraints provided by concurrent MRI. Last, we briefly discuss how various data combinations that the instrument could facilitate, including tissue perfusion, can enable more comprehensive assessment of lesion physiology. ๑ The Authors. Published by SPIE under a Creative Commons Attribution 4.0 Unported License. Distribution or reproduction of this work in whole or in part requires full attribution of the original publication, including its DOI. [DOI: 10.1117/1.JBO.24.5.051409]
\end{abstract}

Keywords: diffuse optics; tomography; breast cancer; multimodality; reconstruction; magnetic resonance imaging.

Paper 180556SSR received Sep. 19, 2018; accepted for publication Dec. 10, 2018; published online Jan. $24,2019$.

\section{Introduction}

Diffuse optical tomography (DOT) is a near-infrared optical imaging technique that provides three-dimensional (3-D) maps of tissue optical and physiological properties in human tissue. ${ }^{1-5}$ The ability of DOT to probe functional tissue parameters noninvasively is particularly attractive for breast cancer imaging, ${ }^{6}$ but the technique faces challenges as well. First, DOT image reconstruction must solve an ill-posed inverse problem; ${ }^{3}$ second, DOT spatial resolution is low due to the diffusive nature of photon transport in tissue. ${ }^{4}$ Importantly, both limitations can be ameliorated by utilizing information from another structural imaging clinical diagnostic. The additional structural information can constrain the DOT reconstruction algorithm and thereby improve assignment of functional tissue properties based on optical spectroscopy. It also offers the possibility of synthesizing composite functional parameters based on DOT and the structural modality, e.g., MRI, X-ray, or ultrasound, that could provide improved diagnosis or prognosis. Thus, there has been a significant effort in the optical community to develop instrumentation that can be combined with other, more standard, clinical imaging modalities.

*Address all correspondence to Jeffrey M. Cochran, E-mail: cochranj@sas .upenn.edu
Indeed, the portable nature, comparatively low cost, and lack of ionizing radiation associated with diffuse optics makes the combination with standard clinical modalities relatively simple compared to the combination of two traditional, stand-alone clinical imaging modalities, such as MRI-PET. To date, several groups have successfully combined DOT with X-ray mammography, ${ }^{7-11}$ ultrasound, ${ }^{12-15}$ or MRI. ${ }^{16-24}$ These groups have demonstrated the clear utility of multimodality DOT instruments. Combining the different imaging modalities into composite instrumentation is arguably the best approach because data from the same coregistered volume can be acquired concurrently. For breast cancer, concurrent imaging is particularly valuable because the compressibility of breast tissue makes coregistration with nonconcurrent data difficult, requiring sophisticated deformation algorithms and assumptions. ${ }^{25}$

To this end, we introduce a hybrid diffuse optical imaging system that operates concurrently with state-of-the-art clinical MRI surface coils in the imaging suite. The instrument acquires both continuous-wave (CW) and time-domain (TD) diffuse optical data in the parallel-plate geometry, permitting both absolute assignment of tissue optical properties and rapid 3-D DOT. The design in the parallel-plate geometry facilitates combining of a sophisticated research-level optical system with standard clinical MRI coils; the latter, in turn, enables improved patient throughput and use of sophisticated and robust MR pulse 


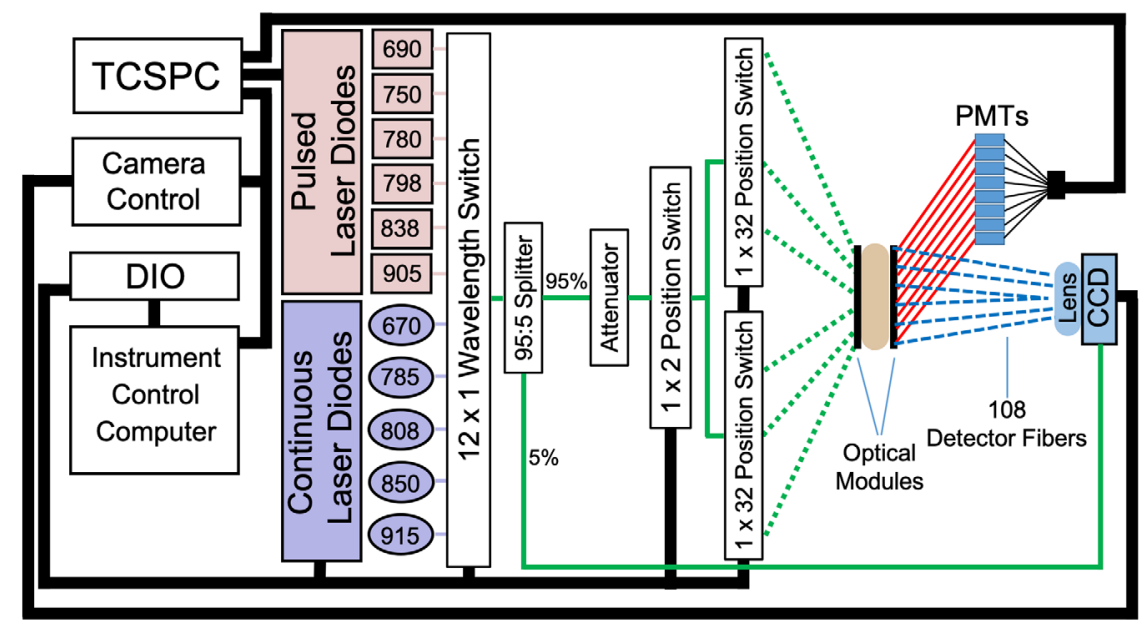

Fig. 1 Schematic of DOT-MRI instrument optics and electronics. This hybrid CW and TD DOT-MRI system employs six short-pulse ( $<70 \mathrm{ps}$ ) laser sources $(690,750,780,798,838$, and $905 \mathrm{~nm})$ for TD measurement of absolute bulk optical properties (absorption and scattering) and five CW laser sources (670, $785,808,850$, and $915 \mathrm{~nm}$ ) for producing 3-D reconstructions of relative optical properties. The sources are coupled to optical imaging modules via a $12 \times 1$ wavelength switch and an effective $1 \times 64$ source position switch, i.e., a $1 \times 2$ switch and two $1 \times 32$ switches. A 95:5 splitter is also used, with the $95 \%$ arm coupled to the source position switch and the $5 \%$ arm coupled to a reference channel. CW detection is performed via detection fibers (108 positions) mounted on a plate and imaged onto a CCD, and TD detection (8 positions) is accomplished with PMTs and TCSPC modules. All components are controlled via the instrument computer, digital input/output channels, and a National Instruments DAQ board.

sequences. Moreover, the instrument can incorporate diffuse correlation spectroscopic measurements (20 source and 20 detector positions) that utilize temporal fluctuations of multiply scattered light intensity to probe tissue blood flow. ${ }^{4,26,27}$ In total, the design thus offers arguably the most versatile and spatially dense optical dataset reported to date for joint optical-MR imaging systems.

At this stage, the joint DOT-MRI instrument has been deployed in the clinic at the Hospital of the University of Pennsylvania. We have begun to measure healthy volunteers to test the patient interface and data acquisition algorithms. The primary purpose of this contribution is to describe the instrumentation in detail and to present an experimental evaluation of its operation and capabilities. Specifically, image reconstructions of a tissue phantom are presented, which demonstrate accurate reconstruction of optical properties and the concrete benefits of spatial constraints provided by the concurrent MRI. We will also briefly discuss how various data combinations that the instrument could facilitate, including tissue perfusion measured by DCS ${ }^{28-33}$ or DCE-MRI, ${ }^{34-36}$ can enable more comprehensive assessment of lesion physiology and biomarkers.

\section{Instrument Design}

This instrument integrates both TD and CW diffuse optical platforms for DOT with either 1.5T or 3T MRI. Adjustable optical modules permit optimized optode positioning for each subject and enable optoelectronic compatibility with both standard clinical systems and future 7T MRI research platforms. As noted previously, this instrument offers the largest in-magnet, i.e., concurrently measured with MRI, DOT spatial data sets reported to date. Acquiring large datasets is particularly difficult in-magnet due to the requirement of a large number of long $(\sim 10 \mathrm{~m})$ optical fibers. Figure 1 displays a schematic of the optical and electronic instrumentation in the system, and
Fig. 2 displays the optoelectronic instrument rack. We will elaborate in detail on various components below.

\subsection{Continuous-Wave Optoelectronic System}

The CW measurements are performed using five steady-state AlGaAs lasers with wavelengths $670 \mathrm{~nm}$ (Sony, Tokyo, Japan), $785 \mathrm{~nm}$ (Frankfurt Laser Company, Friedrichsdorf, Germany), $808 \mathrm{~nm}, 850 \mathrm{~nm}$, and $915 \mathrm{~nm}$ (Roithner LaserTechnik-Vienna, Austria). The typical output power of the laser sources at the tissue interface is between 10 and $27 \mathrm{~mW}$. These lasers feed five of the inputs to a $12 \times 1$ optical switch (PiezoJena, Jena, Germany), the output of which is coupled to a 95:5 optical fiber splitter (OZ Optics - Carp, Ontario, Canada). The 95\% output of the splitter is then fiber-coupled to an attenuator, which is coupled to a $1 \times 2$ optical switch (DiCon FiberOptics, Richmond, California), and then further to two $1 \times 32$ optical switches (DiCon FiberOptics). For CW laser sources, the attenuator is set to maximize signal delivered to the tissue. The source fibers are housed in 10-m long, flat 48-strand optical cables (Molex, Inc., Lisle, Illinois). The proximal ends are broken out into FC connectors, which attach to the 64 source switch outputs. The distal ends are coupled via an MTP connector and broken out into fibers, which are inserted into the optical modules discussed in Sec. 2.3.

$\mathrm{CW}$ detection is performed using $1081-\mathrm{mm}$ diameter optical fibers (FiberOptic Systems, Inc., Simi Valley, California), whose proximal ends are mounted to an imaging plate and imaged onto an Andor IKon CCD (Model No.: DW936N-\#BV-Belfast, Northern Ireland, United Kingdom). The 5\% output from the fiber splitter coupled to the $12 \times 1$ switch is also mounted onto this imaging plate. This fiber serves as a reference channel by which fluctuations in the laser amplitude are directly measured and are thus accounted for via normalization of the detected signal. 


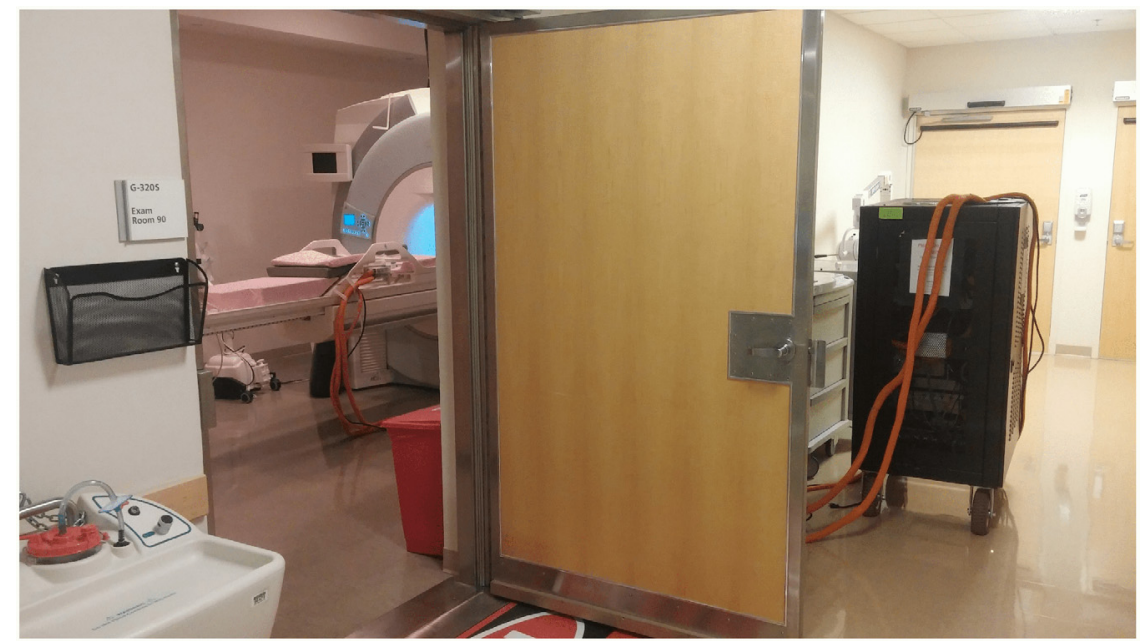

Fig. 2 DOT-MRI system in the clinical MR suite. The optical and electronic components of the DOT-MRI instrument are housed in an instrument rack on pneumatic wheels for easy transport to and from the MR suite. The rack contains all laser sources, the pulsed laser diode driver, all optical switches, the CW detection fiber imaging plate and CCD, PMTs and PMT control-units for TD detection, and TCSPC control instrumentation. When in use, the DOT instrument rack is brought to the control room of the MR suite at the Hospital of the University of Pennsylvania. The fiber bundles then pass into the MRI bore and are incorporated into the clinical breast biopsy RF coil, on which the subject can lie in a prone position to be simultaneously imaged by MRI and DOT.

\subsection{Time-Domain Optoelectronic System}

The TD system consists of six pulsed laser sources (PicoQuant, Berlin, Germany) with wavelengths 690, 750, 780, 798, 838, and $905 \mathrm{~nm}$, coupled to a laser diode driver oscillator (Sepia-II, PicoQuant). These sources feed six of the remaining channels of the $12 \times 1$ wavelength switch (PiezoJena), i.e., channels that are not occupied by the continuous wave sources. These lasers deliver $\sim 30 \mu \mathrm{W}$ average power to the tissue.

TD light is collected by eight 3-mm optical fibers, which are each coupled to a photomultiplier tube (PMT, H7422-50mod, Hamamatsu, Japan). The larger fiber diameter enables more photons to be collected and thus provides a vital improvement in signal-to-noise for the PMT detection system. The instrument contains 16 distinct PMT modules, enabling potential future expansion. When in use, these PMTs are protected from saturation by a source-side attenuator, which can adjust for variation in optical attenuation between subjects. When not in use, individual computer-controlled shutters protect the detectors. The PMTs are connected to two independent Becker \& Hickl (Berlin, Germany) time-correlated single-photon counting (TCSPC) modules (SPC-130). Single-photon counting techniques are necessary to temporally resolve the detected signal collected by the PMTs. Thus, the point-spread functions necessary for the determination of optical properties via TD techniques can be measured.

\subsection{Optical Modules, RF Coil, and Imaging Platform}

All fibers, including the source fibers, the CCD-coupled CW detection fibers, and the PMT-coupled TD detection fibers are gathered together into $\sim 10$-m long mechanically shielded fiber bundles. The bundles need to be $10 \mathrm{~m}$ in length to enable optical data to be collected inside an MR suite; the instrument's electronic modules are not designed to operate in high magnetic fields and must be kept sufficiently far away, i.e., outside the restricted area of high magnetic field. The proximal ends of all fibers are permanently mounted inside four custom-built optical modules made of gray PVC material with a 3-D printed plastic shield on the nontissue side to protect the optical fibers. These materials were demonstrated to have no detectable magnetic susceptibility that could contaminate the MR signal. Each of the four module faces, which are placed in contact with the tissue, has a length of $16.4 \mathrm{~cm}$ and a height of either 3 or $3.5 \mathrm{~cm}$. This provides a sufficient coverage area of source and detector positions for most breasts. The two source modules each contain 32 source fibers. The first detector module contains $54 \mathrm{CW}$ detection fibers and 5 TD detection fibers while the second detector module contains $54 \mathrm{CW}$ detection fibers but only $3 \mathrm{TD}$ detection fibers. The fiber optic modules also contain 20 DCS source fibers, shared across the two detector modules, and 20 single-mode DCS detector fibers (Molex, Inc.), spread across the two source modules. Note that these fibers are not coupled to DCS source and detector instrumentation on the current optoelectronics rack; however, a DCS laser source and detection hardware, i.e., avalanche photodiodes, have been integrated in previous work ${ }^{31}$ and could easily be incorporated into the system in the future. Figure 3 contains photographs of the mounted fibers and the tissue interfaces of the optical modules.

These modules are mounted in a parallel-plate geometry within the windows of two clinically standard Sentinelle RF biopsy coils (INVIVO Corp. Gainesville, FL Part No.:4000044-11). The two source modules are located on the medial side of the subject's breast, and the two detector modules are placed on the lateral side of the breast (see Fig. 3). This alignment puts the source positions farther away from the lateral side of the breast, where a majority of breast tumors form, ${ }^{37}$ and thus limits contamination between the tumor contrast and the known imaging artifacts found near the source plane. ${ }^{38,39}$ In clinical use, the RF coils have open windows with plastic grids so that MRI-guided breast biopsies can be performed. If the plastic grids are removed, the coil provides a window for the optical modules to couple to tissue. Importantly, the addition of these optical modules does not 
(a)

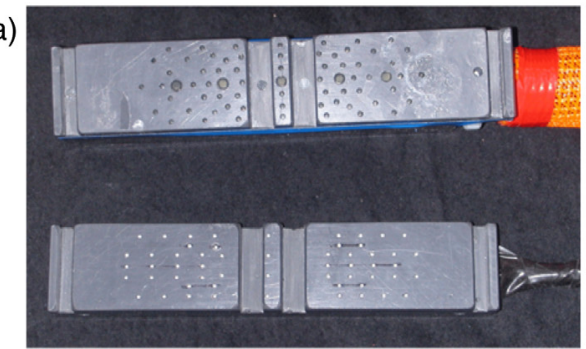

(b)

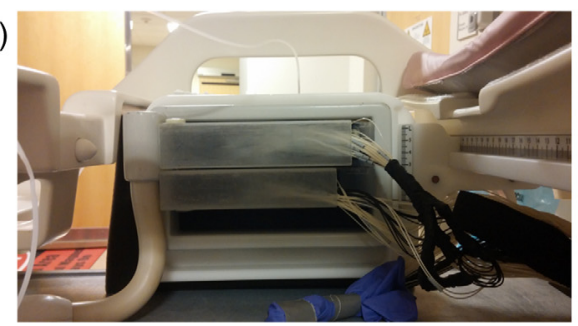

(c)

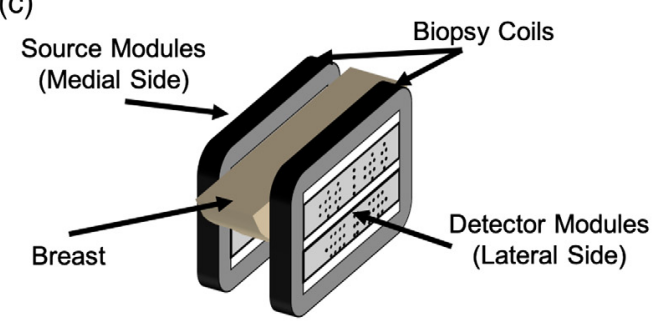

Fig. 3 Optical source and detector modules. (a) Front view of one detector module (top) and one source module (bottom). On the detector module, the large fiber tips are the 3-mm fibers coupled to PMTs, and the smaller tips are either the 1 -mm fibers imaged onto the CCD or DCS source fibers. The fiber tips on the source modules are either source fibers coupled to the optical position switch or single-mode DCS detection fibers. (b) A rear view of the source modules in the breast biopsy coil. (c) Schematic of the optical modules within the breast biopsy coil windows in a sagittal parallel-plate configuration on both sides of the breast. Note that the source modules (medial side) and the detector modules (lateral side) can be moved vertically within the biopsy window to ensure coverage of the tumor region of the breast.

require reconfiguration or modification of the clinical imaging system. The biopsy coils are housed in a patient imaging platform in which the patient lies prone, and the breast is inserted between the two coils for simultaneous optical and MR imaging. The compatible coils available at the Hospital of the University of Pennsylvania can be used in either $1.5 \mathrm{~T}$ or $3 \mathrm{~T}$ large-bore, clinical MR scanners, e.g., the 1.5T Siemens Espree system (Erlangen, Germany) currently used for clinical breast imaging at the Hospital of the University of Pennsylvania. Finally, MRI fiducial markers are mounted on the optical modules to permit coregistration of the DOT and MR imaging geometries.

One key advantage of this instrument is the modularity of the optics at the tissue interface. The number and position of optical modules within the biopsy window can be easily modified, enabling optimization of the source and detector locations. This capability provides better coverage of the tumor region and limits the number of sources and detectors that are not directly in contact with tissue due to the curvature of the breast. In conjunction with the instrument control software, the number of acquired source-detector pairs can also be chosen to optimize spatial resolution (many source-detector pairs) or temporal resolution (few source-detector pairs).

\subsection{System Control and Stability}

All of the aforementioned optical and electrical components, along with the instrument control computer, are housed in a rack-mounted instrument with pneumatic wheels for simple, safe transport of the instrument to and from the MR suite. As previously mentioned, the $\sim 10-\mathrm{m}$ fiber bundles can then be passed into the MR suite. In this way, the optical modules can be mounted on the RF coil while the instrument rack remains outside the high magnetic field of the MRI (see Fig. 2). The instrument computer controls the function of all components through LabVIEW (National Instruments, Austin, Texas) and Becker \& Hickl (Berlin, Germany) software; it is coupled to the optoelectronic components via digital input/output channels, a measurement computing board, and a National Instruments DAQ board (Austin, Texas).

Prior to imaging, the lasers must be turned on for a 30-min warm-up period. After warmup, the $\mathrm{CW}$ laser intensities are stable to $<1 \%$; the remaining power variation is normalized using the directly measured intensity in the low power arm of the 95:5 splitter. The TD system requires a somewhat longer period of $\sim 1$ h to stabilize.

Light leakage at the detectors is minimized to the point that DOT images can be successfully carried out in typical clinical lighting conditions. However, the bore light in the MRI is turned off, and the subject is covered with a sheet to minimize stray light at the patient interface. Additionally, absorbing foam cut-outs are placed underneath the breast to prevent light from passing directly from source fibers to detectors outside the tissue and to reduce artifacts due to the boundary between diffusive and nondiffusive regions.

\section{Data Acquisition and Analysis}

When using the instrument, three imaging sequences must be performed for each subject or phantom measurement: CWDOT, TD-DOS, and MRI (see Fig. 4). Because the optical measurements do not interfere with the magnetic field, the MRI and optical sequences can be performed simultaneously; however, the CW and TD diffuse optical imaging must be performed sequentially. In addition to the target measurement, a reference phantom is imaged using the same TD and CW sequence as the target. This reference measurement enables difference reconstruction, i.e., measuring the differences in optical signal between tissue and a phantom, which reduces error due to coupling coefficients between fibers and tissue.

\subsection{MRI Measurements}

The MRI pulse sequences performed with this instrument are the standard clinical gadolinium-contrast (Gd-contrast) uptake sequences used for breast MR imaging. These sequences typically consist of a series of T1- and T2-weighted structural image sequences, a baseline T1-weighted sequence sensitive to the Gd-contrast agent, and then a series of images with the same Gd-sensitive sequence to capture the temporal uptake dynamics of the Gd-contrast agent. These MR images provide the structure of the breast and a clear subtraction Gd-contrast image of the tumor location. The MRI will also image the fiducial markers placed at known positions on the optical modules, 


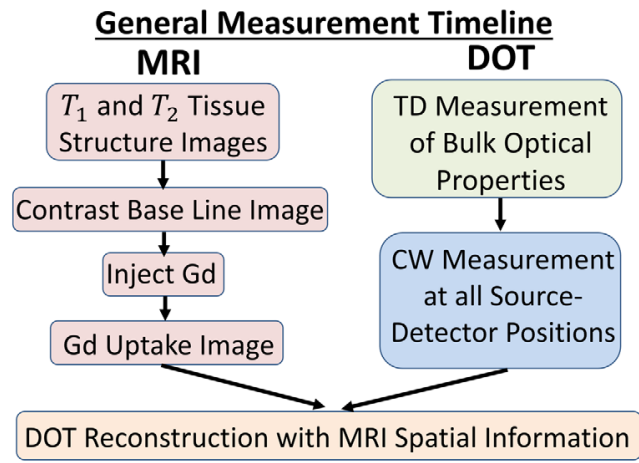

Fig. 4 DOT-MRI imaging sequence flow chart. The DOT-MRI instrument performs the optical and MRI measurements simultaneously. Structural T1- and T2-weighted MRI measurements are made prior to the injection of a gadolinium contrast agent. After the contrast injection, another set of MR images are acquired, thereby permitting subtraction imaging that enables optimal tumor contrast. Simultaneously, optical data are acquired. First, TD measurements are made at several positions to determine absolute bulk optical properties. Then, $\mathrm{CW}$ measurements are made at all source and detector positions to enable full 3-D reconstruction.

which enables the coregistration of the optical and MRI coordinate systems.

\subsection{Time-Domain Optical Measurements}

The optical imaging sequence begins with a series of TD measurements. Approximately 4 of the 64 source positions are illuminated with each of the 6 available pulsed laser wavelengths. These measurements are performed sequentially for each source-wavelength combination, and all eight available PMT detector locations are active for every measurement. In practice, fewer than eight detectors will be used for each source position due to signal limitations caused by differences in sourcedetector separations, which can vary from $\sim 6 \mathrm{~cm}$ to $\sim 12 \mathrm{~cm}$. The PMTs, in conjunction with the TCSPC electronics (see Sec. 2.2), then record point-spread functions for each wavelength/source-detector combination. These point-spread functions are dependent on the absorption and reduced scattering coefficients of the tissue. The light pulse input to the tissue is not a true delta function due to the intrinsic duration of the laser pulse, as well as the broadening of the light pulse that occurs in the optical switches, attenuators, and fibers. This broadening, known as the instrument response function (IRF), is of particular concern with this DOT-MRI instrument due to the requirement of long $(\sim 10 \mathrm{~cm})$ optical fibers on both the source and detector sides. Thus, to distinguish the temporal broadening due to diffusion through tissue from the inherent broadening in the instrument, the IRF must be measured. With this system, the IRF can be found by performing TD measurements without an optically thick diffusive medium, e.g., without a breast or phantom, between the source and detector planes. Care must be taken in this step to attenuate the source light to avoid saturating and/or damaging the PMTs. Once the IRF is known, it serves as an effective temporal source profile, which can be convolved with the Green's function solution for diffuse light propagation in an infinite slab. To calculate the optimal absorption and reduced scattering coefficients, the measured point-spread function is then fit to this convolution of the IRF and the Green's function solution using a nonlinear fitting algorithm, in this case, the MATLAB file exchange function fminsearchbnd. ${ }^{40}$ This method is described in detail in previous publications. ${ }^{16,41}$

Since only a few source positions are used for the TD measurements, the fitted absorption and reduced scattering coefficients for each source-detector pair are typically averaged with all other measurements at the same wavelength, each of which probe different regions of the breast. Performing TD measurements at more source positions could enable a sort of coarse imaging of absolute optical properties; however, this benefit must be balanced by the time it adds to the patient imaging sequence. With this instrument, TD imaging of this type is not feasible within the context of typical MR patient time-windows of less than $1 \mathrm{~h}$.

\subsection{Continuous-Wave Optical Imaging}

The CW imaging sequence is performed after the completion of the TD measurements. Each of the $5 \mathrm{CW}$ laser wavelengths and 63 of the available 64 source positions are cycled through sequentially. One source position is usually left disconnected to provide a sham measurement, with no source illumination, for background normalization purposes. A single frame is acquired by the CCD for each source-wavelength combination. This frame will contain the outputs from the fiber tips at the imaging plate. Thus, each detector fiber will be imaged over an approximate circle containing $\sim 80$ pixels. The detector locations on the CCD are determined using a mask for the sum over all measured frames. The signal used for analysis of a single detector is the sum over all pixels assigned to that detector fiber for a single camera frame. Each detector measurement is then ratio-normalized using the relative intensity of the CCD reference channel to ameliorate signal contamination due to laser intensity fluctuations. The entire sequence of source and wavelength combinations is also performed several times to assess potential motion artifacts or other systematic instabilities. The data from these multiple passes through the entire sequence are then averaged for each wavelength-source-detector combination. In the case of a clear artifact, data are excluded from the average.

An optical contrast agent, indocyanine green, could also be utilized to enhance tumor imaging ${ }^{42-44}$ and track contrast uptake dynamics. In such cases, temporal resolution would take precedence over spectral resolution, and thus, the CW imaging would be performed at only a single wavelength near the peak absorption of the contrast agent.

Once this data is collected, single-wavelength or multispectral reconstruction is performed. The absolute bulk absorption and reduced scattering coefficients from the TD analysis are used as the initial conditions for the reconstruction. Because the high-spatial density system is CW, only the absorption coefficient, and by extension, the chromophore concentrations are reconstructed while the reduced scattering is kept constant. The knowledge of absolute background absorption coefficient from the TD instrumentation transforms the relative absorption coefficients typically reconstructed in CW DOT to absolute values. In principle, multispectral reconstruction, using a Mielike scattering framework and incorporating the TD-measured reduced scattering, could also be used to reconstruct the reduced scattering coefficient.

For image reconstruction, we employ a finite-difference reconstruction method performed using TOAST $++{ }^{45}$ This methodology is a nonlinear, iterative approach, which assumes a small perturbation between the optical properties of the breast 
tissue and a reference phantom and utilizes the ratio of the intensity between the tissue and phantom measurements as the signal in order to normalize for the source-detector coupling coefficients. In the phantom reconstructions presented here, single-wavelength reconstructions are performed. However, for future human subject data, multispectral reconstruction algorithms that directly determine the oxy- and deoxyhemoglobin concentrations could be utilized. Given the substantial number and range of available wavelengths, water and/or lipid concentrations could also be reconstructed in those cases. This task would likely be made easier if a hard-prior constraint were used (see Sec. 3.4), thereby minimizing the under-determined nature of the DOT inverse problem.

\subsection{Incorporation of MRI Data into DOT Reconstructions}

The primary purpose of simultaneous DOT and MRI, other than cross-validation, is to exploit the MRI structural images as a prior constraint for the DOT reconstruction. To this end, after coregistration of the two co-ordinate systems using the MR fiducial markers, the MR images must be segmented into distinct regions. A simple mask for this purpose is created via signal thresholding, in which signal cutoff values between various regions are determined, enabling the creation of a four-region mask, e.g., nontissue, adipose, fibroglandular, and tumor using either the T1- or T2-weighted MR images. Here, for simplicity, the MR images were segmented into nontissue, background breast tissue, and tumor regions using the contrast-enhanced MRI subtraction image, which provides distinct contrast between the tumor and the rest of the breast tissue. Figure 5 contains an example of a phantom MRI and the segmented regions. Note that the regions outside the breast are not reconstructed from the optical measurements.
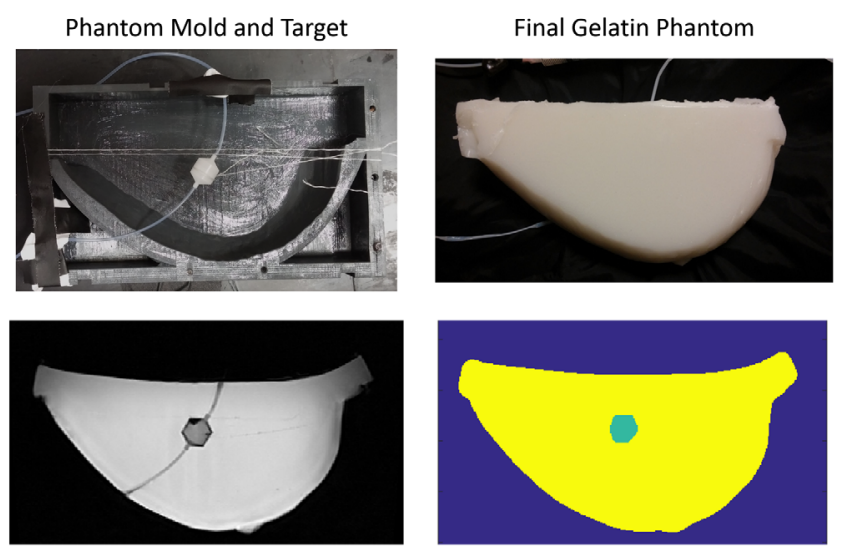

T1-Weighted MRI

3-Region Mask

Fig. 5 Gelatin phantom and mold. (a) One half of the breast-shaped gelatin phantom mold with target, prior to pouring of the gelatin mixture. The target is held in place by thin, white string which does not significantly change the optical properties of the phantom. (b) Final gelatin phantom. Different liquid phantom materials can be injected, via the nylon tubing, into the target within the gelatin. (c) T1-weighted MR image of the phantom. (d) Signal-threshold segmentation of MR image to define distinct regions in the tissue phantom. Here, the image is segmented into a three region-types, i.e., the liquid target, the gelatin phantom, and the background region. A three-region segmented image mask is then ready for use as a hard- or soft-prior constraint for DOT.
Once the MRI has been segmented into distinct regions, these regions can be used to constrain the DOT reconstruction. One can utilize the MRI as either a hard prior or a soft prior for the DOT reconstruction.

A hard-prior constraint uses the segmented image to define distinct spatial regions and then forces the reconstructed optical properties to be homogeneous within each region. This very substantially reduces the number of parameters that must be reconstructed. Hard-prior constraints have been shown to improve quantitative contrast between tumor and normal regions. ${ }^{46-48}$ However, this technique is very sensitive to systematic inaccuracies in the location of the defined regions. ${ }^{49,50}$ Therefore, accurate segmentation and spatial coregistration between modalities is imperative.

Another implementation of the segmented image is as a soft boundary prior, in which the regularization hyperparameter is lower near region boundaries than in the region interior. This enforces a smoothing condition within a given region while allowing for significant updates at the boundaries, where there is more potential for a physiological nonlinearity. Soft-prior regularization has been shown to improve optical contrast relative to unconstrained DOT ${ }^{39,51-53}$ but is generally not as effective in this regard as hard-prior constraints. ${ }^{5,55}$ It also does not reduce the parameter space for the inverse problem since all voxels are still reconstructed. However, soft-prior regularization does have the advantage of being less critically dependent on accurate segmentation.

\section{Tissue Phantom Imaging Tests}

Initial evaluation of the imaging capabilities of the joint DOT-MRI instrumentation was conducted using a tissue-like phantom with both magnetic and optical absorption contrast. This phantom provided a test for the DOT acquisition sequence, data processing, and image reconstruction techniques in a controlled environment with known optical properties.

\subsection{Phantom Construction and Characteristics}

Most diffuse optics phantom experiments utilize liquid phantoms composed of water, ink for absorption contrast, and Intralipid for scattering contrast. This approach is not an ideal solution for experiments performed in the MR suite because liquid phantoms expose expensive MRI equipment to potential damaging spills. Thus, a custom gelatin phantom was created for this investigation.

First, a mold was designed and 3-D printed using a segmented MR image to mimic the approximate shape of a breast. A porcine gelatin powder (Sigma-Aldrich, St. Louis, Missouri) was mixed with water, India Ink (Higgins, Leeds, Massachusetts), a 20\% Intralipid soy emulsion (Fresenius Kabi Global, Bad Homburg, Germany), and a Gd-chelate magnetic contrast agent (MultiHance-Bracco Diagnostics, Milan, Italy). To prevent nondiffusive regions from forming in the phantom, air bubbles were removed from the gelatin mixture using a vacuum pump prior to the gelatin setting. A hollow target, $16 \mathrm{~mm}$ in diameter with $1-\mathrm{mm}$ thick polyoxymethylene walls ${ }^{56}$ was suspended approximately in the center of the gelatin mold prior to the pouring of the gelatin. This target was secured with thin white threads and connected to thin nylon tubing with both ends of the tubing terminating outside of the gelatin phantom (see Fig. 5); this set-up enabled injection of small amounts of liquid with different magnetic and absorption 
properties into the target to create varying levels of contrast with respect to the background phantom.

In this experiment, the background gelatin phantom, which consisted of gelatin, Gd, ink, and Intralipid, had optical properties of $\mu_{\mathrm{a}}=0.05 \mathrm{~cm}^{-1}$ and $\mu_{\mathrm{s}}^{\prime}=8 \mathrm{~cm}^{-1}$ at $785 \mathrm{~nm}$. The concentration of India ink in the target solution was adjusted to provide $4: 1$ optical contrast in $\mu_{\mathrm{a}}$ at $785 \mathrm{~nm}$. Phantom optical properties at other wavelengths can be found in Fig. 7. The background phantom also contained $0.5 \mathrm{mM}$ MultiHance Gd-chelate to create an MR contrast relative to the target, which did not contain the Gd-contrast agent. A reference phantom was also created for the difference measurements by injecting a liquid phantom with the same optical and magnetic properties as the gelatin into the target.

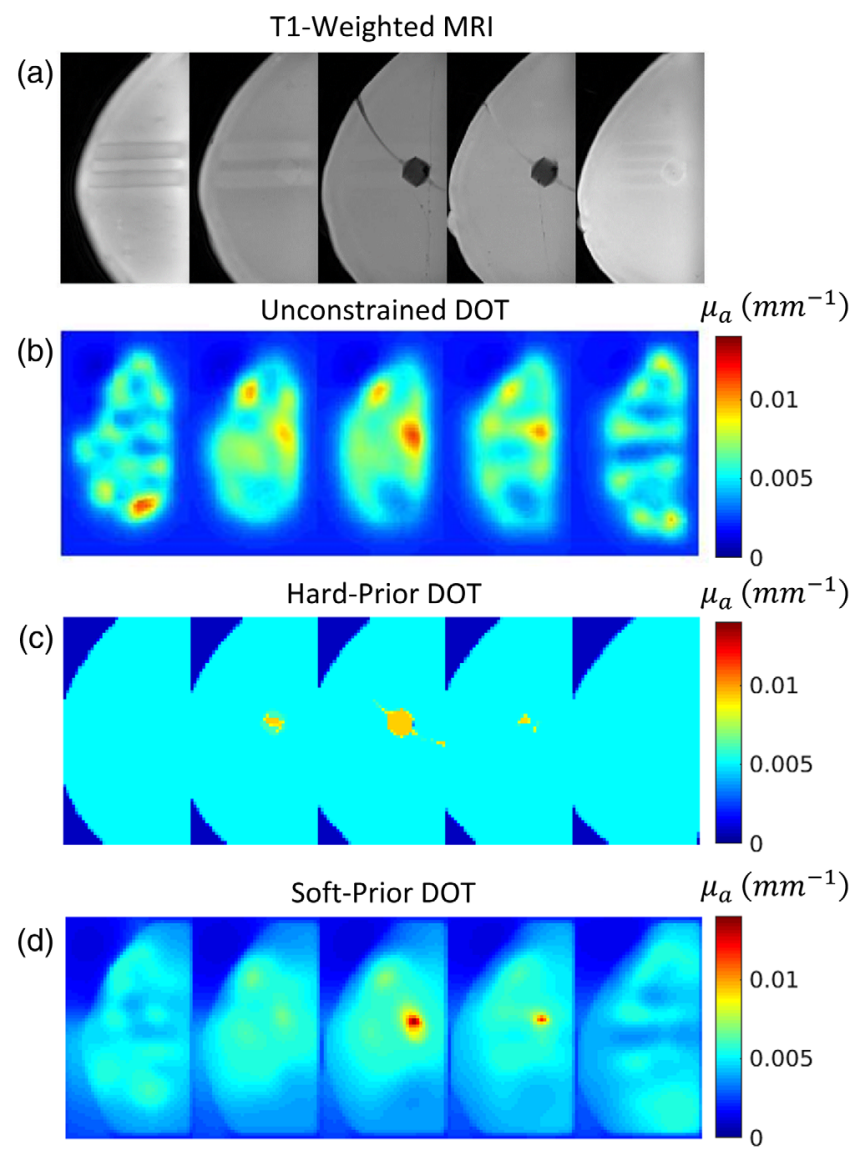

Fig. 6 Unconstrained, hard-prior, and soft-prior DOT reconstructions. (a) Sagittal slices, separated by $1 \mathrm{~cm}$, of a T1-weighted fat-suppressed gradient echo MRI of the gelatin phantom and target. Note that the horizontal lines on the first (source-side) and last (detectorside) slices are indentations in the phantom from the optical modules. (b) Sagittal slices of an unconstrained DOT reconstruction. The DOT reconstruction accurately localizes the target and provides good absorption contrast relative to the background. However, numerous boundary artifacts also arise with reconstructed $\mu_{\mathrm{a}}$ values similar to the targets. (c) Sagittal slices of a DOT reconstruction constrained by a hard spatial prior. Interestingly, although the artifacts are removed by the hard-prior constraint, the contrast here is also reduced. This effect could be due to slight inaccuracies in the co-registration of the segmented MR image. (d) Sagittal slices of a DOT reconstruction constrained by a soft spatial prior. Notice that the contrast is slightly improved and the artifacts are significantly reduced relative to the unconstrained reconstruction.

\subsection{MR and Optical Imaging}

A T1-weighted fat-suppressed gradient echo MRI pulse sequence was run to derive MR images with good contrast between the target and background phantom [see Fig. 6(a)]. Optical data were collected using both the CW and TD measurement systems. A single wavelength reconstruction of the $785 \mathrm{~nm}$ data is presented here. Note that the absolute optical properties measured at $780 \mathrm{~nm}$ with the TD system were assumed to be the same as the optical properties at $785 \mathrm{~nm}$ because the India ink has a weak wavelength-dependence in this spectral regime. Here, the fitted TD data gave absolute bulk optical properties of $\mu_{\mathrm{a}}=0.053 \mathrm{~cm}^{-1}$ and $\mu_{\mathrm{s}}^{\prime}=7.6 \mathrm{~cm}^{-1}$, which are within $6 \%$ and $5 \%$, respectively, of the background values we anticipated in constructing the tissue phantom.

The DOT images were reconstructed using the TOAST++ software suite. ${ }^{45}$ A Gauss-Newton method for the optical property update and total variation regularization were used for the unconstrained reconstruction. ${ }^{57}$ Figure 6(b) shows this unconstrained DOT image, which was created with no a priori information from the MRI. Note that this reconstruction provides relatively good quantitative contrast between the target and background (see Table 1); however, image artifacts of comparable contrast also arise near the tissue phantom boundary and near the source and detector planes.

A priori information from the segmented MRI was used to ameliorate the artifacts and improve contrast. In one case, a hard-prior constraint from MRI was placed on the DOT reconstruction, enforcing homogeneous optical property updates within both the target and background regions [see Fig. 6(c)]. Though this technique eliminates the artifacts, the contrast between the target and background is unexpectedly low. This is likely due to an inaccuracy in the co-ordinate system coregistration and thus imperfect spatial designations for the two regions. To overcome this flaw, soft-prior constraint based on MRI was also utilized. Here, a first-order Tikhonov regularization scheme was used with a regularization parameter that increased with increasing distance away from the backgroundtarget boundary, which smooths the reconstructed parameters at locations away from the known discontinuity between the

Table 1 DOT-MRI reconstructed optical properties. Expected and reconstructed $\mu_{\mathrm{a}}$ for the spatially unconstrained, hard-prior, and soft-prior DOT reconstructions in both the background and target regions, as well as the bulk absolute optical property measurement by the TD system. Note that the reconstructed contrast is unlikely to ever fully reach the expected $4: 1$ value, which represents the ratio of target to background $\mu_{\mathrm{a}}$, due to the broadening of the reconstructed target. Thus, unconstrained and soft-prior reconstructions both demonstrate reasonably good contrast.

$$
\mu_{\mathrm{a}} \text { at } 785 \mathrm{~nm}\left(\mathrm{~cm}^{-1}\right)
$$

Average background Maximum target Contrast

\begin{tabular}{lccc}
\hline Expected & 0.05 & 0.20 & $4: 1$ \\
TD & 0.053 & - & - \\
Unconstrained & 0.06 & 0.14 & $2.3: 1$ \\
Hard-prior & 0.051 & 0.095 & $1.9: 1$ \\
Soft-prior & 0.055 & 0.15 & $2.7: 1$ \\
\hline
\end{tabular}


background and the target. Figure 6(d) displays this soft-prior reconstruction. The boundary artifacts present in the unconstrained reconstruction are now eliminated, and the contrast between the target and background has slightly increased. The soft-prior technique was also used to reconstruct the absorption coefficient at all CW wavelengths, except for $915 \mathrm{~nm}$. The very high water content of the phantom led to increased attenuation of light with wavelengths longer than $900 \mathrm{~nm}$, which prevented reconstruction with significant target-to-background contrast. However, the other wavelengths all provided reasonable contrast (Fig. 7).

\section{Discussion}

\subsection{DOT Reconstruction Validation}

The images reconstructed from a breast-shaped, gelatin phantom discussed in Sec. 4.2 provide a validation of the ability of the DOT-MRI platform to provide high spatial resolution, 3-D images of the absorption coefficient and demonstrate the utility of using MR images as spatial priors. First, the TD system produced accurate quantification of the average absolute absorption and reduced scattering coefficients with errors of only $6 \%$ and $5 \%$, respectively, at $780 \mathrm{~nm}$ and similar accuracy for all other wavelengths. Moreover, the unconstrained CW reconstruction (see Fig. 6), which was performed with no input from the MR image, reconstructed the target with reasonably good contrast $(2.3: 1$ versus an expected contrast of $4: 1$ at $785 \mathrm{~nm}$ ) at the appropriate location in three dimensions. Note that reconstructed images are expected to have lower maximum contrast than the actual physical contrast due to the partial volume effect and spatial broadening of the target, which spreads the total contrast over a larger volume. ${ }^{39,58}$ Thus, this image offers evidence that the high-spatial density DOT system provides quality reconstructions without a priori information. Despite the quality of the localization, however, there were artifacts in the absorption reconstruction near the boundaries, a common issue with DOT reconstructions. ${ }^{59,60}$
To address these artifacts, a priori structural information from the MRI was used. A hard-prior reconstruction, whereby the background and target regions determined by the segmented MRI are forced to update homogeneously, unexpectedly provided worse contrast $(1.9: 1$ at $785 \mathrm{~nm})$ than the unconstrained reconstruction. Inaccuracies of the coregistration of the optical and MRI co-ordinate systems could be the cause of this relatively low contrast. Hard-prior reconstructions are particularly sensitive to this type of image offset. ${ }^{49,50}$ To mitigate this source of error in future measurements, more well-defined fiducial markers in precisely measured locations have been added to this instrument. The soft-prior constraint reconstruction provided the best overall image quality for this phantom, with the highest target-to-background contrast $(2.7: 1)$ and the elimination of the boundary artifacts found in the unconstrained reconstruction.

In future human subject imaging, all three types of reconstruction (unconstrained, hard-prior, and soft-prior) could be used. The unconstrained image is most useful for directly validating DOT as an imaging technology against spatially coregistered, gold-standard MRIs. While the hard-prior is the most commonly used constraint in this type of imaging, we have learned that soft-prior reconstructions offer some distinct advantages. For example, incorporating information about the normal tissue heterogeneity has been shown to be beneficial, both for distinguishing malignant from healthy tissue ${ }^{61}$ and for predicting response to chemotherapy. ${ }^{62,63}$ This information is accessible from a soft-prior reconstruction but is suppressed by a hard-prior constraint.

\subsection{Instrument Advances and Applications}

The primary advances associated with the joint imaging system are the high-spatial density optical information, absolute background optical property quantification via the TD system, compatibility with a clinical RF coil, the inclusion of DCS fibers for measurement of blood flow, and modular optics to optimize data collection. These instrument characteristics help to address

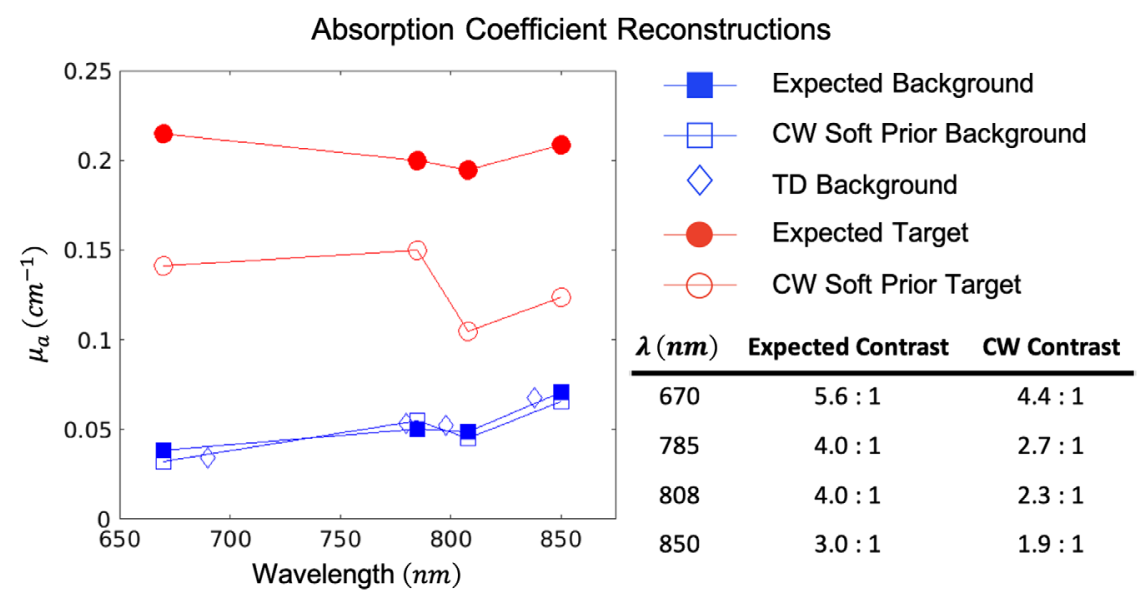

Fig. 7 Absorption coefficient reconstructions. TD-DOS was employed to reconstruct the average absolute background $\mu_{\mathrm{a}}$. Soft-prior constrained CW-DOT was used to reconstruct both background and target $\mu_{\mathrm{a}}$ using the reconstructed $\mu_{\mathrm{s}}^{\prime}$ value from the TD analysis. Note, the TD and CW background $\mu_{\mathrm{a}}$ are very close to the expected values. Clear contrast between the target and background is apparent at all wavelengths; the reduced contrast relative to the true value is expected to arise because of the spatial broadening of the reconstructed target. TD measurements at $905 \mathrm{~nm}$ and $\mathrm{CW}$ data at $915 \mathrm{~nm}$ were not reconstructed here because the higher water content of the phantom attenuated the signal too much at these wavelengths to produce quality reconstructions. 
some of the most interesting applications of DOT in breast cancer. Specifically, improved reconstruction quality over DOT-MRI systems with fewer source and detector positions, and those with only relative optical property calculation, could enhance both the localization and quantification of tumor optical and physiological properties, and allow for the development of composite optical-MR biomarkers. This could enable improved differentiation between malignant and healthy tissue and improved characterization of tumors for prediction of response to chemotherapy. The spatial information provided by the MRI will also improve the identification of tumor and normal tissue regions. The advantages provided by increased spatial information ${ }^{38,39,64,65}$ and a priori localization ${ }^{11,47-51}$ are well-known and have been previously demonstrated by several other groups.

The incorporation of the clinical coil, which has been done previously, ${ }^{66}$ is also a benefit of this system. Effectively, it provides a more robust MR interface and a more comfortable patient experience than a custom-built RF coil, which is more prevalent in multimodality DOT imaging. Patient comfort is very important, not only for the increased ability to recruit study subjects, but also because of the reduction in motion artifacts associated with a comfortable resting position. The modular design of the optical interface with the RF coil also enables optimization of the source and detector locations, individually optimizing coverage of each tumor and enabling subjects with unusually positioned tumors to be enrolled in trials. Additionally, the modularity of the optics enables potential expansion of the instrument, i.e., the introduction of more source and detector fibers, to perform concurrent bilateral breast measurement.

The more robust clinical coil also enables relatively simple implementation of more advanced DCE-MRI techniques, such as K-space weighted image contrast, which relies on fast, compressed-sensing pulse sequences and imparts information about tumor perfusion. ${ }^{34-36}$ These techniques, along with the 20 DCS source fibers and 20 DCS detector fibers, provide two methods of obtaining 3-D maps of blood flow with precise structural knowledge. This blood flow information could also be combined with tissue oxygen saturation measurements, from DOT, to explore oxygen metabolism, ${ }^{6,29,33}$ which represents a relatively new biomarker for exploring cancer physiology.

\subsection{Future Improvements}

During this instrument's initial testing phase, it became apparent that several minor improvements were needed. The first, which has already been completed, is the incorporation of more precise fiducial markers to improve the coregistration between the optical and MRI coordinate systems. The fiducial makers are thin tubes, three of which are attached to each optical module in orthogonal orientations to ensure accurate localization in all three dimensions. A second instrumental shortcoming is the lack of sensitivity of the PMT detectors to the 905-nm pulsed laser, which reduces the number of available TD wavelengths and inhibits the system's ability to accurately reconstruct water concentration. This limitation could be ameliorated by complementing the current PMTs with newer, custom-built PMT modules ${ }^{67}$ that are more sensitive to infrared light. The final significant instrument deficiency was an aspect of the control software. The system is currently designed to capture frames from the CCD with a single exposure time across all wavelengths. Because the various $\mathrm{CW}$ lasers have different powers, and because tissue absorbs some wavelengths more than others, an optimal exposure time for one wavelength could result in saturation or low signal for another wavelength. In the current setup, this flaw can be overcome by manually changing the exposure time for each wavelength in the series; however, this is a time-consuming process which cannot feasibly be done during a patient measurement. Thus, it would be beneficial to automate this procedure by incorporating an exposure-time optimization scheme. An algorithm to accomplish this goal has been designed and is currently being tested for the instrument.

\section{Conclusion}

A joint DOT-MRI platform for simultaneous clinical MRI and hybrid CW and TD optical imaging has been presented. This system has 64 source fiber positions, $108 \mathrm{CW}$ detector fiber positions, and $8 \mathrm{TD}$ detector positions, which represents the most spatially dense DOT-MRI system developed to date. Importantly, these optode positions are contained in adjustable modules that can be coupled to $1.5 \mathrm{~T}$ or $3 \mathrm{~T}$ clinical MR suites, enabling improved patient comfort and the use of more advanced MR pulse sequences. The instrument also utilizes five $\mathrm{CW}$ and six TD laser sources at wavelengths ranging from 670 to $915 \mathrm{~nm}$, which enables absolute quantification of optical properties and tissue chromophore concentrations. Finally, the optical interfaces also contain 20 DCS source fibers and 20 single-mode DCS detector fibers for the potential calculation of 3-D blood flow images.

The DOT reconstruction algorithm has been validated using a breast-shaped gelatin phantom with a liquid target. Unconstrained, soft-prior constrained, and hard-prior constrained images were produced, which demonstrated the ability of the system to accurately locate lesions and provide reasonable levels of target-to-background contrast. The soft-prior constrained image, in particular, provided a quality reconstruction with high contrast and limited artifacts.

This system will be used in the clinic to more accurately quantify tumor and healthy tissue for diagnosis of malignant lesions, prediction of response to neoadjuvant chemotherapy, and exploration of blood flow and oxygen metabolism in breast cancer. Thus, this system provides an excellent opportunity to improve the accuracy of diffuse optical measurements and explore the development of multimodal flow and oxygenation biomarkers that could illuminate tumor physiology.

\section{Disclosures}

D.R.B. and A.G.Y. have pending or granted patents on certain aspects of technology related to diffuse optical spectroscopy. Neither author currently receives income from nor holds equity in a company licensing these patents.

\section{Acknowledgments}

Funding for this work was provided by National Institutes of Health under Grant Nos. R01-EB002109, P41-RR002305, P41-EB014893, NTROI 1U54CA105480, NHLBI HL007915, the Thrasher Research Fund, and the Whitaker and Fulbright programs at the Institute for International Education. The authors would like to thank Dr. Thomas Connick for his assistance in designing and testing the system in the MRI environment, Norman Butler for his generous advice for developing a clinically deployable system, Michael Carman for his assistance in mechanical design, and the MR imaging technicians 
who accommodated our work amidst their busy clinical schedule. We also thank Dr. Soren D. Konecky and Dr. Regine Choe for their assistance in developing patient interfaces and instrument designs, as well as our research coordinators: Madeline Winters, Ellen Foster, Alexandra Vandegrift, Sarah Grundy, and Michelle Wong. This project builds on initial work done by Dr. Xavier Intes and Dr. Vasilis Ntziachristos; the authors appreciate their guidance. Finally, we thank Dr. Britton Chance for his vision and foundational work in the application of diffuse optics to breast cancer.

\section{References}

1. D. R. Leff et al., "Diffuse optical imaging of the healthy and diseased breast: a systematic review," Breast Cancer Res. Trans. 108(1), 9-22 (2008).

2. B. J. Tromberg et al., "Assessing the future of diffuse optical imaging technologies for breast cancer management," Med. Phys. 35(6), 24432451 (2008).

3. S. R. Arridge and J. C. Schotland, "Optical tomography: forward and inverse problems," Inverse Probl. 25(12), 123010 (2009).

4. T. Durduran et al., "Diffuse optics for tissue monitoring and tomography," Rep. Prog. Phys. 73(7), 076701 (2010).

5. D. Grosenick et al., "Review of optical breast imaging and spectroscopy," J. Biomed. Opt. 21(9), 091311 (2016).

6. D. R. Busch et al., "Towards non-invasive characterization of breast cancer and cancer metabolism with diffuse optics," PET Clin. 8(3345-365) (2013).

7. A. Li et al., "Tomographic optical breast imaging guided by threedimensional mammography," Appl. Opt. 42(25), 5181-5190 (2003).

8. Q. Zhang et al., "Coregistered tomographic $\mathrm{x}$-ray and optical breast imaging: initial results," J. Biomed. Opt. 10(2), 024033 (2005).

9. Q. Fang et al., "Combined optical and X-ray tomosynthesis breast imaging," Radiology 258(1), 89-97 (2011).

10. K. E. Michaelsen et al., "Calibration and optimization of 3D digital breast tomosynthesis guided near infrared spectral tomography," Biomed. Opt. Express 6(12), 4981-4991 (2015).

11. B. B. Zimmermann et al., "Multimodal breast cancer imaging using coregistered dynamic diffuse optical tomography and digital breast tomosynthesis," J. Biomed. Opt. 22(4), 046008 (2017).

12. Q. Zhu et al., "Imager that combines near-infrared diffusive light and ultrasound," Opt. Lett. 24, 1050-1052 (1999).

13. M. J. Holboke et al., "Three-dimensional diffuse optical mammography with ultrasound localization in a human subject," J. Biomed. Opt. 5, 237-247 (2000).

14. Q. Zhu et al., "Utilizing optical tomography with ultrasound localization to image heterogeneous hemoglobin distribution in large breast cancers," Neoplasia 7(3), 263-270 (2005).

15. Q. Zhu, S. Tannenbaum, and S. H. Kurtzman, "Optical tomography with ultrasound localization for breast cancer diagnosis and treatment monitoring," Surg. Oncol. Clin. N Am. 16, 307-321 (2007).

16. V. Ntziachristos, X. H. Ma, and B. Chance, "Time-correlated single photon counting imager for simultaneous magnetic resonance and nearinfrared mammography," Rev. Sci. Instrum. 69(12), 4221-4233 (1998).

17. V. Ntziachristos et al., "MRI-guided diffuse optical spectroscopy of malignant and benign breast lesions," Neoplasia 4, 347-354 (2002).

18. B. Brooksby et al., "Magnetic resonance-guided near-infrared tomography of the breast," Rev. Sci. Instrum. 75, 5262-5270 (2004).

19. D. Hsiang et al., "Coregistration of dynamic contrast enhanced MRI and broadband diffuse optical spectroscopy for characterizing breast cancer," Technol. Cancer Res. Trans. 4(5), 549-558 (2005).

20. X. Zhang, V. Toronov, and A. Webb, "Simultaneous integrated diffuse optical tomography and functional magnetic resonance imaging of the human brain," Opt. Express 13(14), 5513-5521 (2005).

21. C. M. Carpenter et al., "Methodology development for three-dimensional MR-guided near infrared spectroscopy of breast tumors," Opt. Express 16(22), 17903-17914 (2008).

22. S. Srinivasan et al., "Imaged guided near-infrared spectroscopy of breast tissue in vivo using boundary element method," J. Biomed. Opt. 15(6), 061703 (2010).
23. F. El-Ghussein et al., "Hybrid photomultiplier tube and photodiode parallel detection array for wideband optical spectroscopy of the breast guided by magnetic resonance imaging," J. Biomed. Opt. 19(1), 011010 (2014).

24. Y. Zhao et al., "Optimization of image reconstruction for magnetic resonance imaging-guided near-infrared diffuse optical spectroscopy in breast," J. Biomed. Opt. 20(5), 056009 (2015).

25. F. S. Azar et al., "Standardized platform for coregistration of nonconcurrent diffuse optical and magnetic resonance breast images obtained in different geometries," J. Biomed. Opt. 12(5), 051902 (2007).

26. T. Durduran et al., "Diffuse optical measurement of blood flow, blood oxygenation, and metabolism in a human brain during sensorimotor cortex activation," Opt. Lett. 29(15), 1766-8 (2004).

27. D. A. Boas et al., "Establishing the diffuse correlation spectroscopy signal relationship with blood flow," Neurophotonics 3(3), 031412 (2016).

28. T. Durduran et al., "Diffuse optical measurement of blood flow in breast tumors," Opt. Lett. 30(21), 2915-2917 (2005).

29. C. Zhou et al., "Diffuse optical monitoring of blood flow and oxygenation in human breast cancer during early stages of neoadjuvant chemotherapy," J. Biomed. Opt. 12(5), 051903 (2007).

30. G. Yu, "Near-infrared diffuse correlation spectroscopy in cancer diagnosis and therapy monitoring," J. Biomed. Opt. 17(1), 010901 (2012).

31. D. R. Busch et al., "Blood flow reduction in breast tissue due to mammographic compression," Acad. Radiol. 21(2), 151-161 (2014).

32. R. Choe et al., "Optically measured microvascular blood flow contrast of malignant breast tumors," PLoS One 9(6), e99683 (2014).

33. J. M. Cochran et al., "Longitudinal optical monitoring of blood flow in breast tumors during neoadjuvant chemotherapy," Phys. Med. Biol. 62(12), 4637-4653 (2017).

34. H. K. Song and L. Dougherty, "K-space weighted image contrast (KWIC) for contrast manipulation in projection reconstruction MRI," Magn. Reson. Med. 44(6), 825-832 (2000).

35. H. K. Song and L. Dougherty, "Dynamic MRI with projection reconstruction and KWIC processing for simultaneous high spatial and temporal resolution," Magn. Reson. Med. 52(4), 815-824 (2004).

36. L. Dougherty et al., "High frame-rate simultaneous bilateral breast DCE-MRI," Magn. Reson. Med. 57(1), 220-225 (2007).

37. S. Rummel et al., "Tumour location within the breast: does tumour site have prognostic ability?," Ecancermedicalscience 9, 552 (2015).

38. J. P. Culver et al., "Three-dimensional diffuse optical tomography in the parallel plane transmission geometry: evaluation of a hybrid frequency domain/continuous wave clinical system for breast imaging," Med. Phys. 30, 235-247 (2003).

39. H. Y. Ban et al., "Heterodyne frequency-domain multispectral diffuse optical tomography of breast cancer in the parallel-plane transmission geometry," Med. Phys. 43(7), 4383-4395 (2016).

40. J. D’Errico, “fminsearchbnd,” Mathworks File Exchange (2012).

41. S. R. Arridge, M. Cope, and D. T. Delpy, "The theoretical basis for the determination of optical pathlengths in tissue: temporal and frequency analysis," Phys. Med. Biol. 37(7), 1531-1560 (1992).

42. V. Ntziachristos et al., "Concurrent MRI and diffuse optical tomography of breast after indocyanine green enhancement," Publ. Natl. Acad. Sci. USA 97, 2767-2772 (2000).

43. A. Corlu et al., "Three-dimensional in vivo fluorescence diffuse optical tomography of breast cancer in humans," Opt. Express 15(11), 66966716 (2007).

44. D. R. Busch, "Computer-aided, multi-modal, and compression diffuse optical studies of breast tissue," PhD Thesis, University of Pennsylvania (2011).

45. M. Schweiger and S. Arridge, "The toast++ software suite for forward and inverse modeling in optical tomography," J. Biomed. Opt. 19(4), 040801 (2014).

46. B. Brooksby et al., "Combining near-infrared tomography and magnetic resonance imaging to study in vivo breast tissue: implementation of a Laplacian-type regularization to incorporate magnetic resonance structure," J. Biomed. Opt. 10(5), 051504 (2005).

47. M. A. Mastanduno et al., "MR-guided near-infrared spectral tomography increases diagnostic performance of breast MRI," Clin. Cancer Res. 21(17), 3906-3912 (2015).

48. J. Feng et al., "Multiobjective guided priors improve the accuracy of near-infrared spectral tomography for breast imaging," J. Biomed. Opt. 21(9), 090506 (2016). 
49. G. Boverman et al., "Quantitative spectroscopic diffuse optical tomography of the breast guided by imperfect a priori structural information," Phys. Med. Biol. 50(17), 3941-3956 (2005).

50. B. Deng et al., "Impact of errors in experimental parameters on reconstructed breast images using diffuse optical tomography," Biomed. Opt. Express 9(3), 1130-1150 (2018).

51. X. Intes et al., "Diffuse optical tomography with physiological and spatial a priori constraints," Phys. Med. Biol. 49(12), N155 (2004).

52. M. Guven et al., "Diffuse optical tomography with a priori anatomical information," Phys. Med. Biol. 50(12), 2837-2858 (2005).

53. P. K. Yalavarthy et al., "Weight-matrix structured regularization provides optimal generalized least-squares estimate in diffuse optical tomography," Med. Phys. 34(6), 2085-2098 (2007).

54. B. W. Pogue et al., "Implicit and explicit prior information in near-infrared spectral imaging: accuracy, quantification and diagnostic value," Philos. Trans. A Math. Phys. Eng. Sci. 369(1955), 4531-4557 (2011)

55. L. Wu et al., "Shape-parameterized diffuse optical tomography holds promise for sensitivity enhancement of fluorescence molecular tomography," Biomed. Opt. Express 5(10), 3640-3659 (2014).

56. R. Ziegler et al., "Nonlinear reconstruction of absorption and fluorescence contrast from measured diffuse transmittance and reflectance of a compressed-breast-simulating phantom," Appl. Opt. 48(24), 4651-4662 (2009).

57. M. Schweiger, S. R. Arridge, and I. Nissila, "Gauss-Newton method for image reconstruction in diffuse optical tomography," Phys. Med. Biol. 50(10), 2365-2386 (2005).

58. M. A. O'Leary, "Imaging with diffuse photon density waves," $\mathrm{PhD}$ Thesis, University of Pennsylvania (1996).

59. B. W. Pogue et al., "Spatially variant regularization improves diffuse optical tomography," Appl. Opt. 38(13), 2950-2961 (1999).

60. R. Choe et al., "Differentiation of benign and malignant breast tumors by in-vivo three-dimensional parallel-plate diffuse optical tomography," J. Biomed. Opt. 14(2), 024020 (2009).

61. D. R. Busch et al., "Computer aided automatic detection of malignant lesions in diffuse optical mammography," Med. Phys. 37(4), 1840-1849 (2010).

62. D. R. Busch et al., "Optical malignancy parameters for monitoring progression of breast cancer neoadjuvant chemotherapy," Biomed. Opt. Express 4(1), 105-21 (2013).

63. J. M. Cochran et al., "Tissue oxygen saturation predicts response to breast cancer neoadjuvant chemotherapy within 10 days of treatment," J. Biomed. Opt. 24(2), 021202 (2018).

64. M. L. Flexman et al., "Digital optical tomography system for dynamic breast imaging," J. Biomed. Opt. 16, 076014 (2011).

65. V. Krishnaswamy et al., "A digital X-ray tomosynthesis coupled near infrared spectral tomography system for dual-modality breast imaging," Opt. Express 20(17), 19125-36 (2012).
66. M. A. Mastanduno et al., "Adaptable near-infrared spectroscopy fiber array for improved coupling to different breast sizes during clinical MR," Acad. Radiol. 21(2), 141-150 (2014).

67. P. Taroni et al., "Seven-wavelength time-resolved optical mammography extending beyond $1000 \mathrm{~nm}$ for breast collagen quantification," Opt. Express 17(18), 15932-15946 (2009).

Jeffrey M. Cochran is a postdoctoral fellow in the Department of Physics and Astronomy at the University of Pennsylvania. He received his $B S$ degree in physics from Denison University and his $\mathrm{PhD}$ in physics from the University of Pennsylvania. His research is focused on developing noninvasive imaging biomarkers of disease using diffuse optical techniques.

David R. Busch is an assistant professor in the Department of Anesthesiology and Pain Management and Department of Neurology and Neurotherapeutics at the University of Texas Southwestern Medical Center. His work focuses on developing noninvasive and minimally invasive optical tools to monitor deep tissues in health and disease, as well as throughout therapy.

Li Lin is currently a PhD candidate at Caltech under the tutelage of professor Lihong V. Wang. He earned his master's degree at the University of Pennsylvania in 2013 and his bachelor's degree at Tianjin University in 2011. His research focuses on photoacoustic computed tomography and microscopy (PAM), specifically in human breast and animal brain imaging.

Simon Arridge received his BA degree in natural sciences from Cambridge University and his PhD in medical physics from University College London. Currently, he is professor of image processing in the Department of Computer Science at University College London, director of the Centre for Inverse Problems, and a member of the board of directors of the Centre for Medical Image Computing. His research interests are in inverse problems in imaging, mathematical modelling, and computational methods.

Arjun G. Yodh is the James M. Skinner professor of science in the Department of Physics and Astronomy at the University of Pennsylvania. He directs the Laboratory for Research on the Structure of Matter, an interdisciplinary materials institute that hosts the Penn MRSEC. His biomedical research is oriented toward diffuse optical imaging and monitoring, aiming to demonstrate the potential of these tools for functional imaging/monitoring in brain, breast, and muscle.

Biographies of the other authors are not available. 